\title{
Green Physical Processing Technologies for the Improvement of Food Quality
}

\author{
Daming Fan (D), ${ }^{1}$ Hui-Min David Wang, ${ }^{2}$ \\ Srinivas Janaswamy, ${ }^{3}$ and George A. Cavender $\mathbb{1}^{4}$ \\ ${ }^{1}$ School of Food Science and Technology, Jiangnan University, Wuxi, China \\ ${ }^{2}$ National Chung Hsing University, Taichung, Taiwan \\ ${ }^{3}$ South Dakota State University, Brookings, SD, USA \\ ${ }^{4}$ University of Georgia, Athens, GA, USA \\ Correspondence should be addressed to Daming Fan; fandm@jiangnan.edu.cn
}

Received 19 December 2017; Accepted 20 December 2017; Published 4 June 2018

Copyright (c) 2018 Daming Fan et al. This is an open access article distributed under the Creative Commons Attribution License, which permits unrestricted use, distribution, and reproduction in any medium, provided the original work is properly cited.

With the rapid developments and burgeoning technologies in the food industry, the green, efficient, and fast-oriented physical processing tools are of immense interest to industrialists and academicians. They indeed reduce the environmental pollution and energy consumption. The pursuit of these novel arsenals is coupled with auxiliary strategies immensely to maintain the food quality attributes such as nutrients, texture, color, and freshness. Furthermore, they influence the food composition and structure, including the flavor, physical properties and functions, and in turn the product quality. However, there is an unmet need to clarify the intrinsic interactions between the food quality and physical processing. At this critical juncture, this special issue provides the required platform for the researchers to showcase their current efforts on the applications of physical processing technologies for improved food applications.

L. Zhang et al. investigate the influence of steaming and cooking on the phenolic content and antioxidant activity of millet and provide a desirable processing means for millet. They further optimized the processing conditions to improve the retention rate of total phenolic content (TPC). D. Tang et al. tackled the optimal conditions of heat treatment (HT) and HT coupled with preservative treatment for Ponkan fruit (Citrus reticulata Blanco cv. Ponkan) storage. Y. Xie et al. evaluate the effect of sodium acid sulfate (SAS) and UV-C treatment on the fresh-cut potatoes during storage. Q. Liang et al. illuminate the effect of low-frequency ultrasound on the enzymolysis of corn protein with the degree of hydrolysis $(\mathrm{DH})$, conversion rate of protein $(\mathrm{CR})$, and $\mathrm{IC}_{50}$ value of $\mathrm{DPPH}$ as analytical indicators. S.-Q. Tian et al. address the effect of sweeping frequency ultrasound pretreatment on the angiotensin converting enzyme (ACE) inhibitory activity of zein hydrolysates and enzymolysis thermodynamics. S. Deng et al. present the high-voltage electric field thawing characteristics of the frozen tofu. L. Chen et al. address the chemical compositions and bioactive substances of Xinjiang maca and evaluate its antifatigue activity using a mouse model. T. Li et al. present the effect of overdrying the potato starches on surimi products, which includes the change of chemical interactions and chemical composition. I. Boublenza et al. compare the physicochemical parameters, antioxidant activity, lipid composition, and sensory analyses of initial and roasted carob pod powder obtained at different roasting temperatures. L.-L. Huang et al. reveal the phenomenon that Feizixiao lychee has the higher density compared to Guiwei lychee and Nuomici lychee. Z. Yin et al. established an HPLC method for simultaneous determination of quercitrin, 3-hydroxy phloridzin, and phloridzin in M. halliana tea. The nutritional compositions were determined in accordance with the relevant national standards and methods. Y. Chen et al. demonstrate the antioxidant activity of five tea extracts with a potential application in cancer treatment. 
We strongly believe that this special issue provides the required impetus in developing novel green and clean processing tools that are economical to address the growing human population demands for well-balanced and healthy foods.

Daming Fan

Hui-Min David Wang Srinivas Janaswamy George A. Cavender 


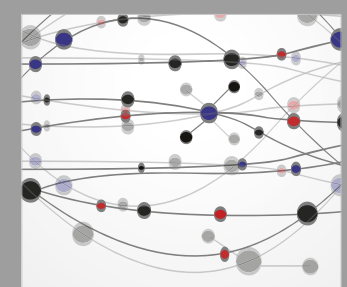

The Scientific World Journal
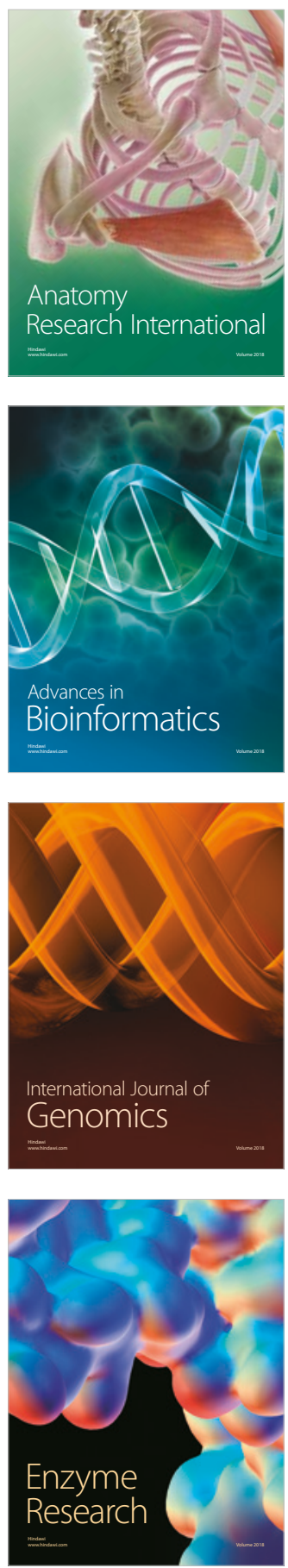
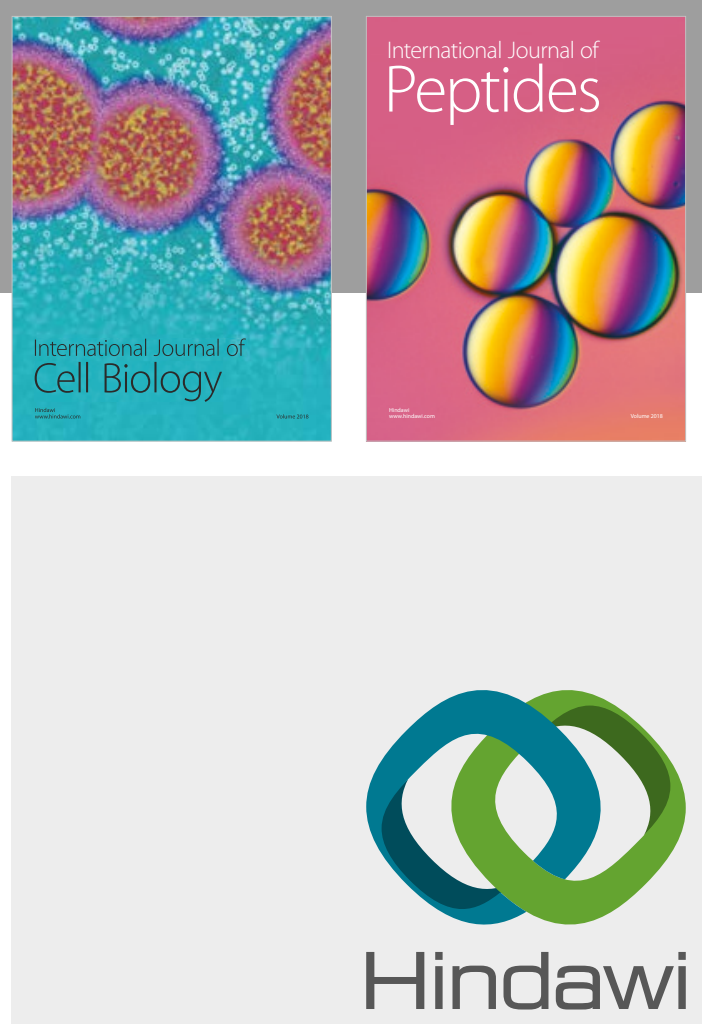

Submit your manuscripts at

www.hindawi.com
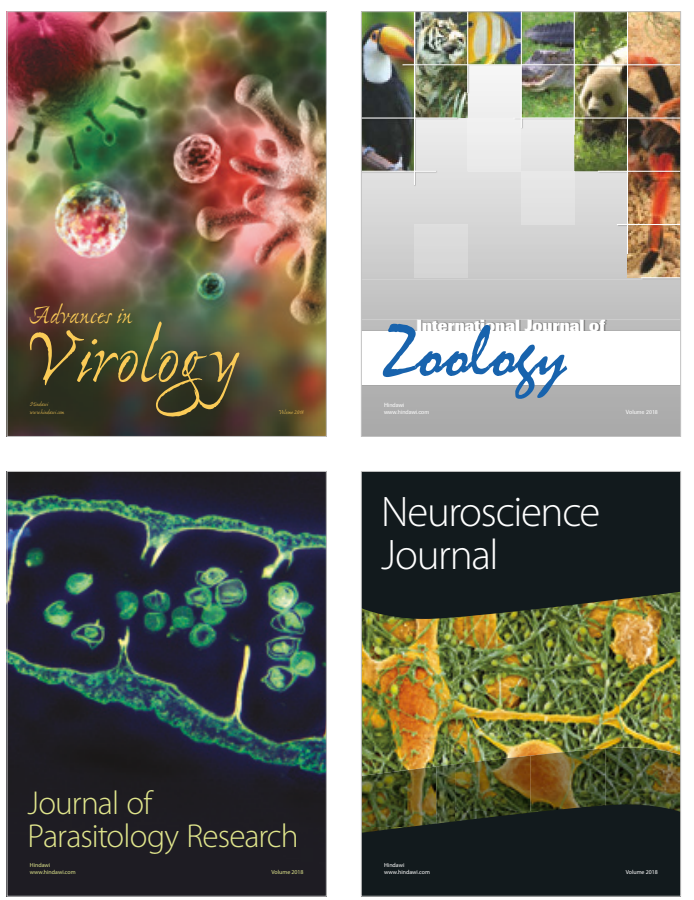
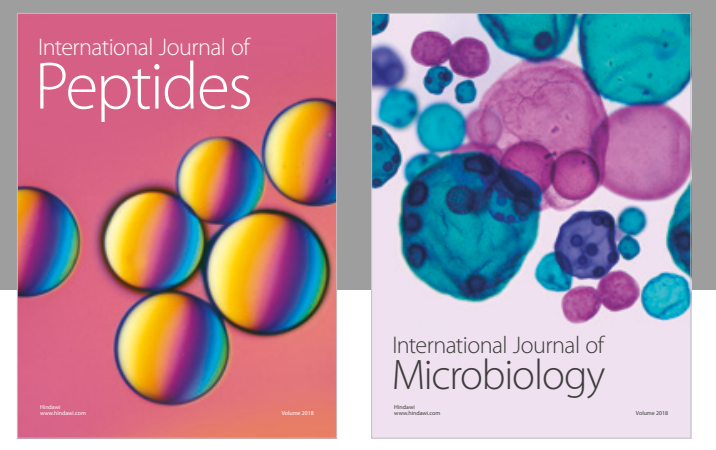

nternational Journal of Microbiology
Journal of
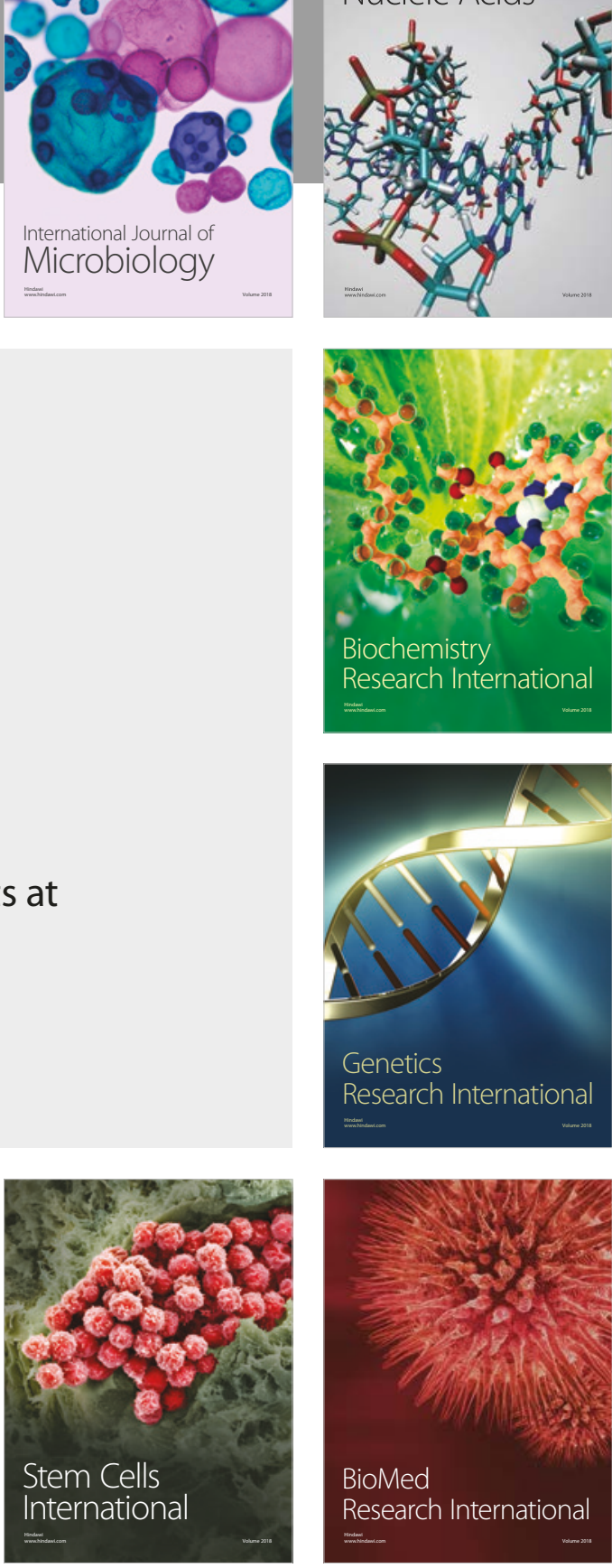
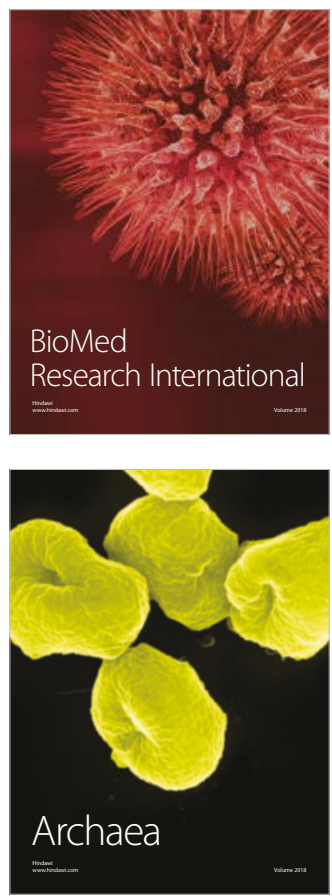